ANZIAM J. 45(2004), 401-422

\title{
MAXIMISING OUTPUT FROM OIL RESERVOIRS WITHOUT WATER BREAKTHROUGH
}

\author{
S. K. LUCAS 1
}

(Received 23 May, 2002; revised 15 May, 2003)

\begin{abstract}
Often in oil reservoirs a layer of water lies under the layer of oil. The suction pressure due to a distribution of oil wells will cause the oil-water interface to rise up towards the wells. Given a particular distribution of oil wells, we are interested in finding the flow rates of each well that maximise the total flow rate without the interface breaking through to the wells. A method for finding optimal flow rates is developed using the Muskat model to approximate the interface height, and a version of the Nelder-Mead simplex method for optimisation. A variety of results are presented, including the perhaps nonintuitive result that it is better to turn off some oil wells when they are sufficiently close to one another.
\end{abstract}

\section{Introduction}

When oil is trapped in a porous rock reservoir by boundaries of impermeable rock, a layer of water is often found underneath (see, for example, Muskat [8], Bear [1]). When oil is removed from the reservoir by an oil well, it will generate a pressure gradient which will also pull the water towards the well. This is counterbalanced by gravity forces due to density differences. As long as the flow rate is not too large, the oil-water interface may reach a stable shape below the well. Pumping too quickly may lead to the undesirable situation of the interface entering the well, and water being pumped to the surface as well as water. This is known as water breakthrough.

A variety of models have been developed over the years, based upon Darcy's law, to model the steady-state shape of the interface. Many of these are referenced or described in Lucas et al. [4], Lucas and Kucera [5] and Gunning et al. [3], although this last paper is more interested in extending to dual completion problems. Zhang and coworkers $[12,13]$ also considered the time-dependent evolution of the interface in a bounded domain, where no steady-state interface shape exists.

\footnotetext{
${ }^{1}$ School of Mathematics and Statistics, University of South Australia, Mawson Lakes, SA 5095 , Australia; e-mail: stephen.lucas@unisa.edu.au.

(C) Australian Mathematical Society 2004, Serial-fee code 1446-1811/04
}

401 


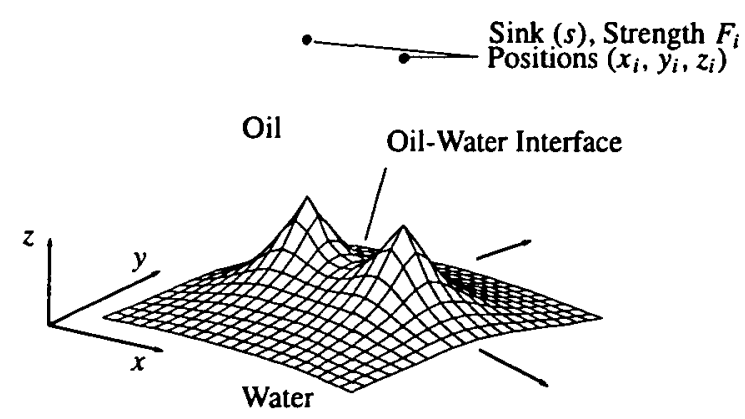

FIGURE 1. The geometry of the water coning model.

In the axisymmetric cases of the work cited above, one particularly important feature discussed is the critical pumping rate - the largest flow rate for an oil well without water breakthrough. While Lucas and Kucera [5] developed a boundary integral formulation to accurately find the interface height given an arbitrary distribution of oil wells, the technique is not appropriate for finding the flow rate from each well that leads to the maximum total flow rate. For a given set of flow rates, the boundary integral method takes hours to find an interface height. Since optimisation software can require hundreds or thousands of iterations to converge, the time required would be prohibitive.

Here, we develop a simple approximate model for quickly finding the position of the oil-water interface under the influence of an arbitrary distribution of oil wells, as well as whether water breakthrough occurs, and use it to find the flow rates at a given distribution of wells that maximises the total flow without water breakthrough. Our motivation for the cases we shall consider has been influenced by the development of horizontal drilling technology. Since points of inflow can be placed anywhere along the well, it is of interest to see what is the best way to distribute them. We begin in Section 2 by deriving the governing equations for flow in porous media with an oil-water interface, as well as the conditions the interface must satisfy. Section 3 develops the Muskat model for approximating the height of the oil-water interface, shows the level of approximation involved, and describes the care required to use the model to predict water breakthrough. Section 4 describes the optimisation technique we use to maximise the total flow, and why it is appropriate for this problem. Section 5 discusses a wide variety of optimal solutions.

\section{Governing equations}

Following closely the derivation in Lucas and Kucera [5], consider as a model for porous rock an isotropic homogeneous medium of constant permeability $k$ which 
occupies all space. Assume the space is filled with either oil or water, and that there is a sharp interface (justified in Bear [1]) between the two fluids, as shown in Figure 1 . We can then apply Darcy's law independently to both regions, where fluid viscosities and densities are different. The oil well(s) are modelled by point $\operatorname{sink}(\mathrm{s})$ within the oil layer.

Assume that the upper fluid (oil) has density $\rho_{1}$ and viscosity $\mu_{1}$, and the lower fluid (water) has density $\rho_{2}\left(>\rho_{1}\right)$ and viscosity $\mu_{2}$. The fluids are separated by an interface denoted by $z=\zeta(x, y, t)$. Darcy's law applied to both fluids gives

$$
\mathbf{u}^{(i)}=-\frac{k}{\mu_{i}} \nabla \hat{p}^{(i)} \quad \text { for } i=1,2,
$$

where

$$
\hat{p}^{(i)}=p^{(i)}+\rho_{i} g z
$$

is the modified pressure, $\mathbf{u}^{(i)}$ is the velocity, $\mu_{i}$ the viscosity, and $\rho_{i}$ the density. The background reservoir pressure is assumed zero. The addition of this term, in any event, is trivial. The incompressibility condition in both fluids is $\nabla \cdot \mathbf{u}^{(i)}=0$, which, when taken with (2.1), gives Laplace's equation for the modified pressures as

$$
\nabla^{2} \hat{p}^{(i)}=0
$$

with the boundary condition that $\mathbf{u}^{(i)}$ and hence $\hat{p}^{(i)}$ tends to zero at infinity. Note that (2.3) for the modified pressure assumes no point sinks. We also have dynamic and material boundary conditions on the interface as respectively

$$
\begin{aligned}
& \text { (a) } p^{(1)}=p^{(2)} \quad \text { and } \\
& \text { (b) } \frac{\partial \zeta}{\partial t}+\mathbf{u} \cdot \nabla(\zeta-z)=0,
\end{aligned}
$$

where $\mathbf{u}$ is the velocity of the interface. Finally, a specification of the sink strength is required. Here, an oil well is modelled as a point sink of volume flow rate $m$. Using the convention that $m>0$ for flow into the sink, and with (2.1), the expression for the suction pressure in an infinite porous medium is

$$
p_{s}=\frac{-m \mu_{1}}{4 \pi k \sqrt{\left(x-x^{\prime}\right)^{2}+\left(y-y^{\prime}\right)^{2}+\left(z-z^{\prime}\right)^{2}}}
$$

where the sink is in the upper fluid at position $\left(x^{\prime}, y^{\prime}, z^{\prime}\right)$. Here we assume that the plane $z=0$ is the position of the oil-water interface far from the sink. Alternatively, it is the position of the oil-water interface if no wells are operating. Since we are considering a steady-state solution, an initial interface shape is not required, and $\partial p / \partial n=0$ can be specified on the interface, as we require it to be static at the steady 
state. This also implies that $\hat{p}^{(2)}=0$, since the steady-state problem requires no flow in the lower fluid. In addition, the kinematic boundary condition (2.4b) becomes identically zero.

Working in the upper fluid, and so dropping the superscript, the form of the dynamic boundary condition (2.4a) using (2.2) is

$$
g \zeta\left(\rho_{2}-\rho_{1}\right)+\hat{p}=0 \quad \text { on } z=\zeta(x, y) .
$$

Note that the steady-state problem implies that $z=\zeta(x, y)$. By scaling lengths with respect to $z^{\prime}$, and pressure with respect to $m_{0} \mu_{1} / k z^{\prime}$, where $m_{0}$ is a typical sink strength, and choosing $m_{0}=\mu_{1} / g z^{2}\left(\rho_{2}-\rho_{1}\right) k$, the dimensionless form of the dynamic boundary condition (2.6) can be rewritten as

$$
\tilde{\zeta}+\tilde{p}=0 \quad \text { on } \tilde{z}=\tilde{\zeta}(\tilde{x}, \tilde{y}),
$$

where $\tilde{\zeta}$ and $\tilde{p}$ are dimensionless interface height and dynamic pressure respectively. This implies that the sink is at the (dimensionless) point $\left(\tilde{x}^{\prime}, \tilde{y}^{\prime}, 1\right)$, and that $(2.5)$ becomes, in dimensionless form,

$$
\tilde{p}_{s}=\frac{-F}{4 \pi \sqrt{\left(\tilde{x}-\tilde{x}^{\prime}\right)^{2}+\left(\tilde{y}-\tilde{y}^{\prime}\right)^{2}+(\tilde{z}-1)^{2}}},
$$

where $F=m \mu_{1} /\left(k z^{\prime 2}\left(\rho_{2}-\rho_{1}\right) g\right)$ is the only dimensionless parameter that appears in the problem, and represents a balance between the suction force of the sink and the gravitational restoring force of the denser fluid. Thus, we are interested in solving

$$
\nabla^{2} p=-F \delta\left(\mathbf{x}-\mathbf{x}^{\prime}\right)
$$

where the tildes have from here on been removed from all dimensionless quantities, and $\delta$ is the Dirac delta function acting at position $\mathbf{x}^{\prime}$ representing an oil well.

Up to now we have assumed that there is a single point sink of strength $m$ at position $\left(x^{\prime}, y^{\prime}, 1\right)$ in the upper (oil) region. The addition of further sinks for a more general model is straightforward. Having $n$ sinks, each of dimensionless sink strength $F_{i}$ at position $\mathbf{x}_{\mathbf{i}}{ }^{\prime}$, replaces $(2.8)$ by

$$
\nabla^{2} p=-\sum_{i=1}^{N} F_{i} \delta\left(\mathbf{x}-\mathbf{x}_{\mathbf{i}}{ }^{\prime}\right)
$$

\section{The Muskat model}

In the work of Lucas et al. [4] and Lucas and Kucera [5], integral equation methods were developed to find the interface shape to high accuracy, both for axisymmetric and 


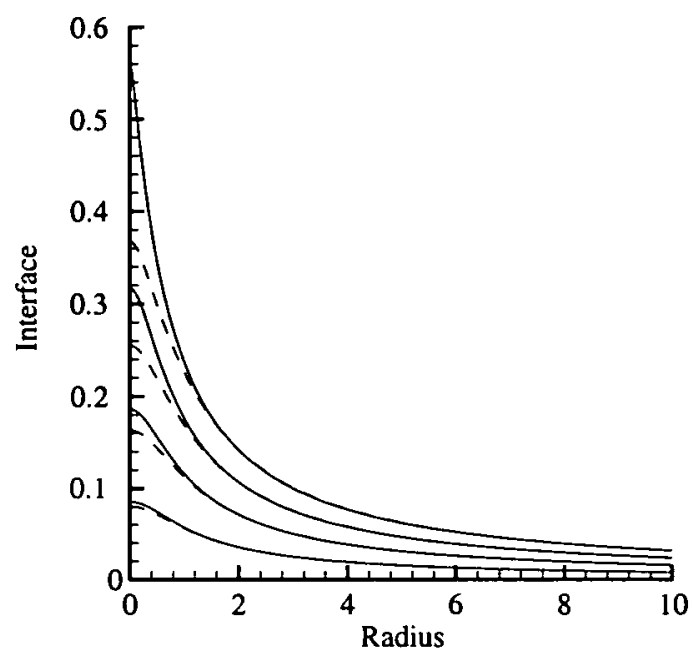

FIGURE 2. Interface heights under the influence of a single well at $(0,0,1)$ of strengths $F=$ $0.5,1.0,1.5,2.0$, where the solid line is the integral equation solution, and the dashed line is due to the Muskat model.

general three-dimensional sink distributions. In the second case, hours of computer time were required to find a solution. The closer the interface is to breakthrough, the longer it takes. Even with the faster machines available today, the time required to find an interface is prohibitive considering how many are required in an optimisation algorithm.

In this paper, we will use the Muskat $[8,9]$ model to approximate the height of the interface. This extremely simple model, developed in the 1930's, balances the suction pressure force with the gravitational force ((2.7)) but calculates the pressure assuming that the interface is flat. In this case, the pressure can be found analytically using the method of images, and (2.7) becomes

$$
\zeta(x, y)=\sum_{i=1}^{n} \frac{F_{i}}{4 \pi}\left[\frac{1}{r_{i}}+\frac{1}{r_{i}^{\prime}}\right],
$$

where

$$
\begin{aligned}
& r_{i}=\sqrt{\left(z_{i}-\zeta(x, y)\right)^{2}+\left(x-x_{i}\right)^{2}+\left(y-y_{i}\right)^{2}}, \\
& r_{i}^{\prime}=\sqrt{\left(z_{i}+\zeta(x, y)\right)^{2}+\left(x-x_{i}\right)^{2}+\left(y-y_{i}\right)^{2}},
\end{aligned}
$$

with $n$ sinks of strength $F_{i}$ at positions $\left(x_{i}, y_{i}, z_{i}\right)$. For each point on the interface $(x, y),(3.1)$ is an algebraic equation that is easily solved using the secant method. In fact, generating enough solutions to find the interface shape at a mesh of points dense enough for good three-dimensional graphics takes about a second on a typical PC. Due to the lack of digital computers in the 1930's, Muskat and Wyckoff [9] solved 


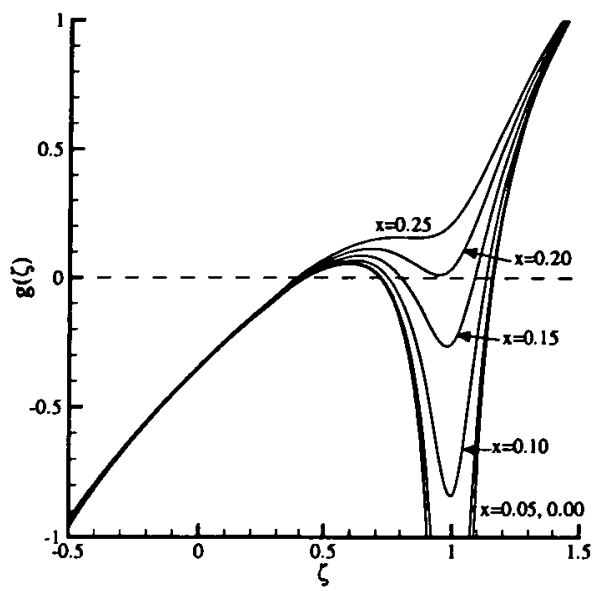

FIGURE 3. $g(\zeta)$ for various values of $x$.

this graphically at selected positions for a single oil well. Blake and Kucera [2] also considered the Muskat model for a single sink.

While appearing deceptively simple, there are two issues of importance when using the Muskat model. They are the fact that this is an approximate model, and that there turn out to be multiple solutions of (3.1) that can cause difficulties. It is these multiple solutions, in fact, that allow us to decide whether an interface exists for a given distribution of oil wells.

3.1. Approximation error The error in the Muskat model is introduced in the approximation of the pressure term. As soon as the interface is not flat, the method of images is no longer applicable. To assess the errors introduced, Figure 2 compares interface solutions for a single sink at $(0,0,1)$ of strengths $F=0.5,1.0,1.5,2.0$ using both the Muskat model and the accurate integral equation solution of Lucas et al. [4]. A similar graph can also be found in Blake and Kucera [2]. We see that the Muskat model underestimates the height of the interface underneath the sink when $F$ is large, and is quite poor for $F \geq 1.5$. However, the Muskat model's performance is more impressive when we consider looking for the largest value of $F$ for which an interface exists. Extensive testing in Lucas and Kucera [5] established that this maximum value is $F=2.050$, with a maximum interface height of 0.661 directly under the sink. The Muskat model has the maximum $F=2.418$ with maximum interface height 0.5774 . This gives an error in the maximum $F$ value of about $18 \%$. Considering the comparison of times between the full integral equation solution and the Muskat solution (hours to under a second), and the fact that the problem is an idealised one anyway (infinite extent, homogeneous medium), we feel that these errors are small enough to justify using the Muskat model. 

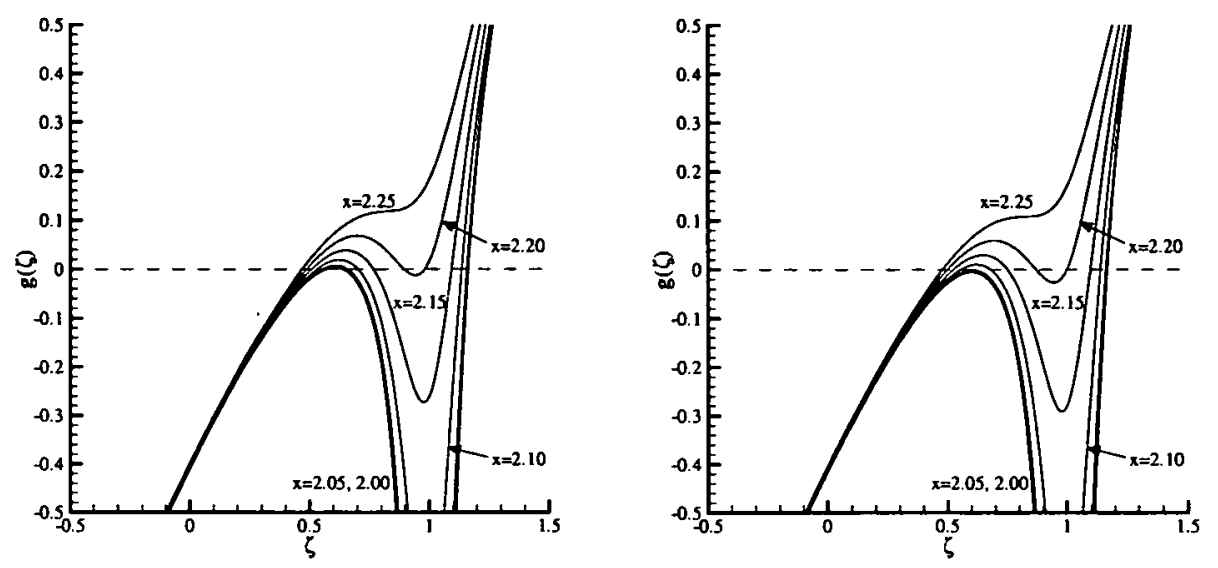

FIGURE 4. $g(\zeta)$ for various values of $x$ for two sinks at $( \pm 2,0,1)$ of strengths 2.08 (left) and 2.11 (right).

3.2. Multiple solutions It turns out that the algebraic equation (3.1) has multiple solutions, and care must be taken in deciding which solution is appropriate, particularly when the interface is near breakthrough. As an example, let us consider the case of a single sink of strength 2.2 at $(0,0,1)$. Applying (3.1) along the line $y=0$ means that the interface height is the solution of

$$
g(\zeta)=\frac{2.2}{4 \pi}\left[\frac{1}{\sqrt{(\zeta-1)^{2}+x^{2}}}+\frac{1}{\sqrt{(\zeta+1)^{2}+x^{2}}}\right]-\zeta=0
$$

Figure 3 shows $g(\zeta)$ for $x=0.00,0.005, \ldots, 0.25$. Remember that here $x$ represents the distance away from directly under the sink. For $x \leq 0.15$, there are three possible solutions to $g(\zeta)=0$. The solution larger than one (the height of the sink) is obviously not physically valid, leaving two possibilities. However, for $x \geq 0.20$, the larger of these solutions disappears, leaving only one valid solution. Since discontinuities are not allowed in the interface, it is the smaller of the solutions of $g(\zeta)=0$ that defines the interface.

While the curves in Figure 3 are for a special case, the form of $g(\zeta)$ for arbitrary distributions of sinks is similar. As another example, consider Figure 4, showing $g(\zeta)$ for two sinks at $( \pm 2,0,1)$ of strengths 2.08 on the left and 2.11 on the right. The area of interest is now near $x=2.00$. As before, there is a solution greater than one which does not correspond to a valid interface, which we ignore. Sufficiently far from under a sink, there is a single solution, which corresponds to the interface height. As we get closer to underneath the sink ( $x=2.20$ here) an additional spurious solution appears. However, what is of most interest in Figure 4 are the curves for $x=2.00,2.05$. On the left the interface and spurious solutions get very close to each other, and we need to be careful that we don't jump from the interface solution manifold to the spurious 
one. On the right, we see that the curves don't quite reach zero, and the only solution is greater than one, which is invalid. If we were following the interface from the far field in to under the sink in this case, the solution would have a sudden jump to above the level of the sinks.

Figure 4 helps us devise a strategy for finding whether an interface exists, and if it does, what shape it takes. We start by finding a solution far from under the sinks, and working in towards the sinks using a uniform mesh. The solution at the previous point (as well as plus $10^{-3}$ ) will be used as the starting points for the secant method to find the solution at the next point. If the secant method doesn't converge, or converges to a solution which is either greater than one or involves a sudden jump, then this is evidence that a stable interface does not exist with this distribution of oil wells. In the work presented here, with accuracies of three decimal places for sink strengths, experiment has shown that a mesh size of 0.005 is adequate, and that the interface shape should be found in a region up to two units away from directly under any sink. It is important to check the entire interface, not just directly under the sinks, because when there are multiple sinks the maximum height of the interface is not necessarily directly under a sink. Finally, in all the cases we investigate here, the sink distributions are such that we only need to find the interface (if it exists) along a line, usually chosen as the $y$ axis.

\section{Finding optimal solutions}

Using the Muskat model with the care described in the previous section, we can quickly decide whether a particular set of sinks with given strengths leads to a stable interface or not. We can now state the optimisation problem of interest:

Given the positions of $n$ sinks, determine the individual sinks strengths $\left\{F_{i}\right\}_{i=1}^{n}$ that maximise $\sum_{i=1}^{n} F_{i}$ with the constraint that a stable interface exists.

We shall call the individual sink strengths at optimal the critical sink strengths. While the function to maximise is extremely simple, standard constrained optimisation techniques such as Kuhn-Tucker leading to SQP are not appropriate. This is because the constraint curve is not known a priori; we instead have a test that returns a yes/no answer on whether a particular set of sinks yields a stable interface or not. In addition, as we shall see later, the boundary of the feasible region (those values of the sink strengths that lead to a stable interface) is not smooth. Some initial attempts using SQP with the equality constraints that the Muskat formula (3.1) is satisfied at select points, and inequality constraints that the interface stays below one (feasible), were somewhat successful. Unfortunately, the difficulty in choosing the right points at which to supply constraints meant that all but the simplest problems were not amenable to analysis by this technique. 
4.1. Method of bisection Since the function we are maximising is linear and the feasible region appears convex (see ahead), the boundary of the feasible region can be found by bisection: given values for the first $n-1 F_{i}$ 's (usually on a mesh), use bisection to find the largest value of $F_{n}$ that has a stable interface to a given precision (typically six digits in this work). For problems with one parameter, we immediately find the optimal solution. With two or more parameters, we can find the boundary of the feasible region, and choose the mesh point with the largest total flow rate. While this approach works, it is increasingly slow as the number of sinks increase. Experience suggests that this method is useful when there are two parameters, but even with three, the amount of effort to find a single optimal solution to any accuracy is excessive.

4.2. The Nelder-Mead downhill simplex method While little used in sophisticated optimisation packages, the Nelder-Mead downhill simplex method (NM method) continues to be competitive in select areas. It is the only "standard" minimisation algorithm that doesn't require a derivative, and while not considered terribly efficient, it is quite robust. The MATLAB [6] routine fminsearch is one standard implementation. Considering the a priori unknown shape of the feasible region for a particular distribution of sinks, as well as its non-smooth character, the NM method is quite appropriate here. It starts with a set of $n+1$ points in $n$ dimensions (a simplex), identifies the worst point, and reflects it through the mean of the others. If it is better, it can be expanded in the given direction. If worse, it can be contracted back, and if still worse, all points can be contracted towards the current best. Convergence is obtained when the range from best to worst points is sufficiently small. The code used to implement the NM method in this work is that of Press et al. [10].

We note at this point that the NM method is a local optimisation method whose convergence is dependent upon the initial guess. Also, it has recently been shown that in some cases the NM method will not converge, as in McKinnon [7]. More complicated variants have been developed (see for example Price et al. [11]) that guarantee convergence. However, none of these difficulties occurred here, and the standard NM method was acceptable.

4.2.1. Constrained Nelder-Mead using a penalty method The NM method is an unconstrained minimisation algorithm, while we have a constrained problem where the constraint boundary is initially unknown. One option is to incorporate a penalty method. Since the optimal solution will be on the boundary of the feasible region, exponential or other smooth penalty functions will not give correct results. A better choice would be a discontinuous penalty, like

$$
\text { Minimise } g-\sum_{i=1}^{n} F_{i}, \quad \text { where } g= \begin{cases}0 & \text { in the feasible region, } \\ 1000 & \text { outside the feasible region. }\end{cases}
$$




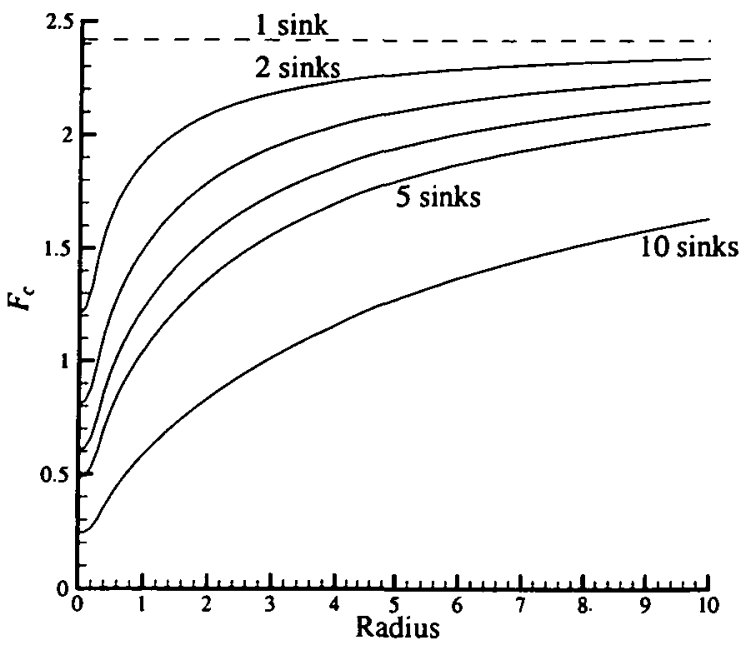

FIGURE 5. Critical sink strengths for $1,2,3,4,5$ and 10 sinks on a circle of a given radius.

One would hope that the derivative-free NM method would be able to cope with such a function. Ideally, the simplex of points would move to the boundary of the feasible region, and move along it to the optimal point. Unfortunately, computational experiments indicate that this method only partially works. Much of the time, the simplex reaches the boundary and cannot find the proper direction easily, and so gets "stuck". Clearly, more sophisticated methods are required.

4.2.2. Nelder-Mead on the feasible boundary Due to the linear nature of the function to maximise, we expect the optimal solution will lie on the boundary of the feasible region. A more productive method is to then only search on this boundary. Our approach is to reduce the dimension of the problem by one: given $F_{1}, F_{2}, \ldots, F_{n-1}$, use bisection to find the value of $F_{n}$ that finds the boundary of the feasible region, then minimise $-\sum_{i=1}^{n} F_{i}$. Since the feasible boundary is non-smooth, the NM method is a good optimisation algorithm to use, and works well in all cases ahead.

4.2.3. Non-negative Nelder-Mead on the feasible boundary Finally, some of the scenarios we will investigate lead to situations where the optimal solution has some sinks of negative strength - they are replaced by sources. Since it is poor practice to pump oil into a reservoir, a non-negative constraint on flow rates is necessary. We simply write $F_{i}=\alpha_{i}^{2}$ for $i=1,2, \ldots, n-1$ and optimise in $\alpha$-space. However, since $F_{n}$ is found on the feasible boundary, there is no guarantee that it will be non-negative. Luckily, the results ahead indicate that sinks on the edges of a group of sinks will not be of negative strength at the optimum, and so we choose the $n$th sink on the edge of the group of sinks. Once again, this approach yields good results. 


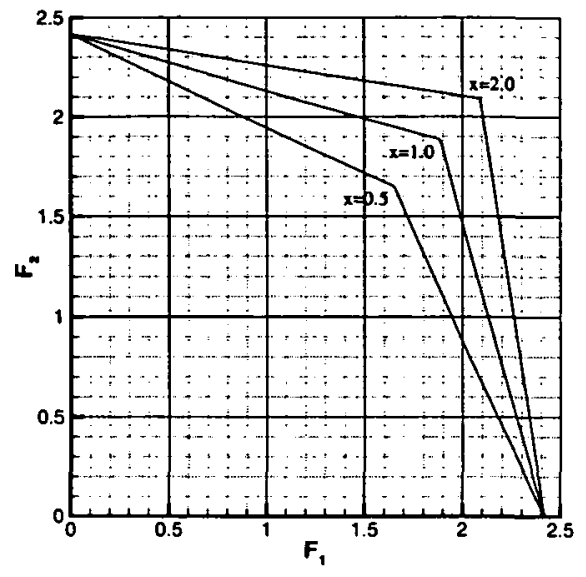

(a)

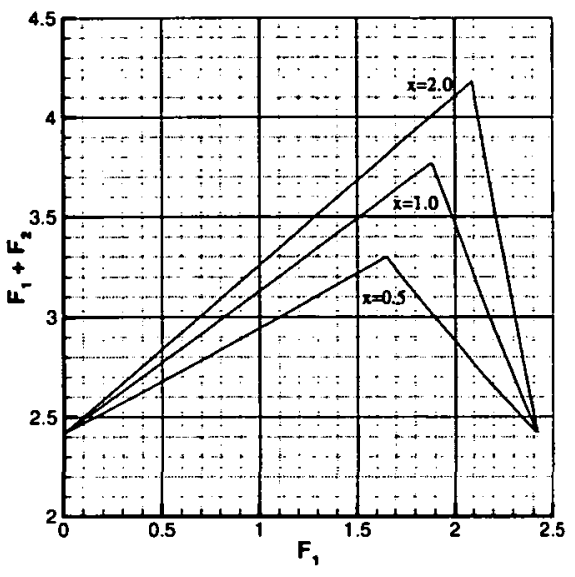

(b)

FIGURE 6. For two sinks of strengths $F_{1}$ at $(-x, 0,1)$ and $F_{2}$ at $(x, 0,1), x=0.5,1,2$, (a) feasible regions and (b) sums of sink strengths along the feasible boundary.

\section{Results and discussion}

5.1. Critical sink strengths - one sink The simplest optimal problem involves $n$ sinks equally spaced around a circle of a given radius. Due to symmetry, all the sinks should be of the same strength, and so we have a one-parameter problem which is easily solved by bisection. Figure 5 shows critical sink strength $F_{c}$ (the largest for which a stable interface exists) versus circle radius for $1,2,3,4,5$ and 10 sinks. The one sink case is the horizontal dashed line at height 2.418 , since the circle radius has no effect when there is only one sink. The multi-sink cases are asymptotic to the single-sink case, although the curves are flattening out as the number of sinks increases. We can also see a turning point in the curves for a radius less than one half. This is due to the fact that when the sinks are sufficiently close together, they essentially act as if they are a single sink. They need to get sufficiently far from each other that the interface maxima move from the centre to more under the sinks for the critical sink strengths to increase more quickly.

5.2. Feasible regions - two sinks While Figure 5 includes solutions for two sinks, it is instructive to look at some feasible regions. Figure 6 (a) shows feasible regions for two sinks of strengths $F_{1}$ at $(-x, 0,1)$ and strength $F_{2}$ at $(x, 0,1)$ for $x=0.5,1,2$. The feasible regions are the area below the relevant lines. While the results could be extended into areas with negative sink strengths, we are limiting ourselves to physically reasonable non-negative sink strengths. The most important feature of these results is that the feasible region boundary is not smooth. With reference to Figure 6 (b), 


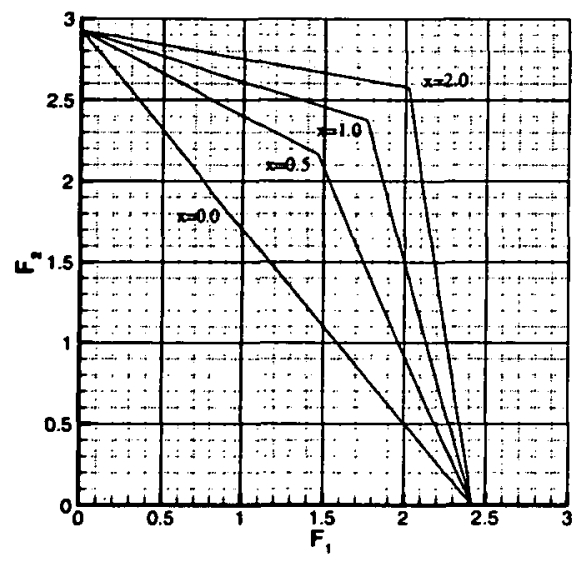

(a)

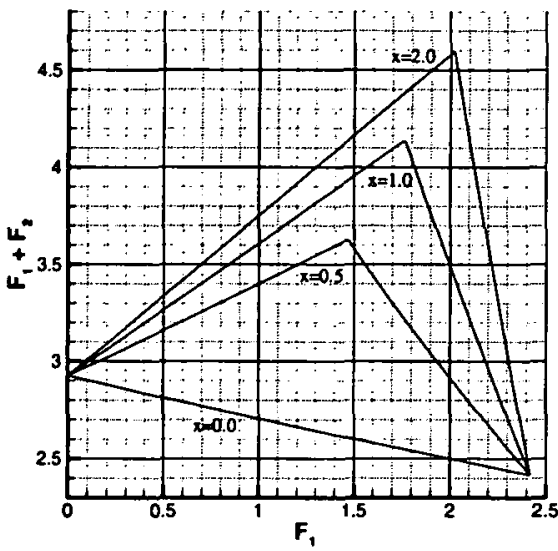

(b)

FIGURE 7. For two sinks of strengths $F_{1}$ at $(-x, 0,1)$ and $F_{2}$ at $(x, 0,1.1), x=0.5,1,2$, (a) feasible regions and (b) sums of sink strengths along the feasible boundary.

which shows the sum of the sinks strengths as a function of $F_{1}$ along the feasible region boundary, we see that the optimum actually lies at this corner. And, it appears that the feasible boundary is made up of straight lines. The astute reader may notice that these feasible regions are very similar to those in linear programming problems, and wonder if the linear programming simplex method would be a very quick and efficient way of approaching this problem. Unfortunately, the main difficulty with this optimisation problem is that we don't know the position of the feasible boundary a priori, and hence corners where we would expect to find the maximum need to be found, hence the methods described in this paper. But it is intriguing that the feasible region of an optimisation problem defined by the existence of a solution to a particular nonlinear equation could be of such an interesting form.

We also include in Figure 7 feasible regions and sums of sink strengths for two sinks of strength $F_{1}$ at $(-x, 0,1)$ and $F_{2}$ at $(x, 0,1.1)$ for $x=0,0.5,1,2$. This is a two-parameter problem, since the right sink is higher than the left one. As expected, this skews the feasible region, and the optimal flow rates have $F_{2}>F_{1}$. We include the $x=0$ case (one sink directly above the other) to show that the best results are obtained when the lower sink is not operating at all. While not shown here, we have found that the lower sink is not necessary for optimal flow when the sinks are sufficiently close $(x<0.2)$.

\subsection{Three sinks}

5.3.1. Equally spaced The next simplest case we will consider involves three sinks in a line. An example feasible region and sums of sink strengths are included in 

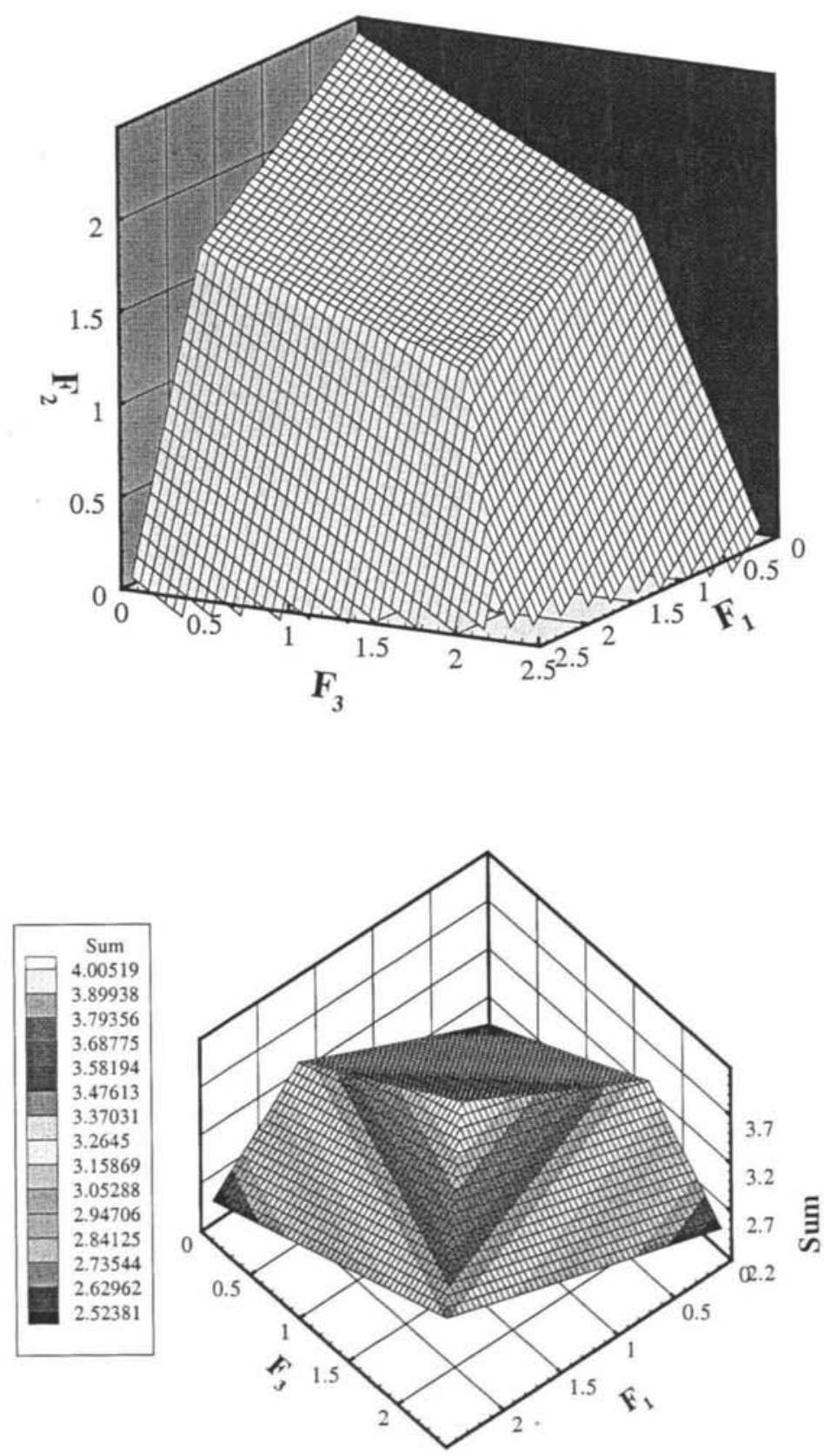

FIGURE 8. Feasible region and sums of sink strengths on the boundary for three sinks of strengths $F_{1}$ at $(-1,0,1), F_{2}$ at $(0,0,1)$ and $F_{3}$ at $(1,0,1)$. 


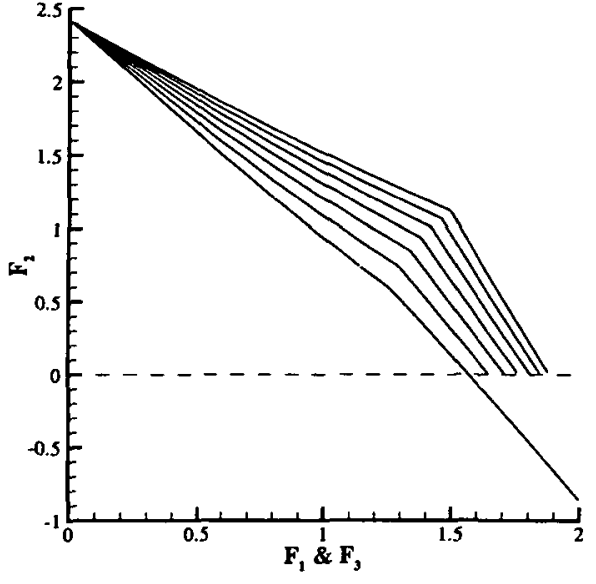

(a)

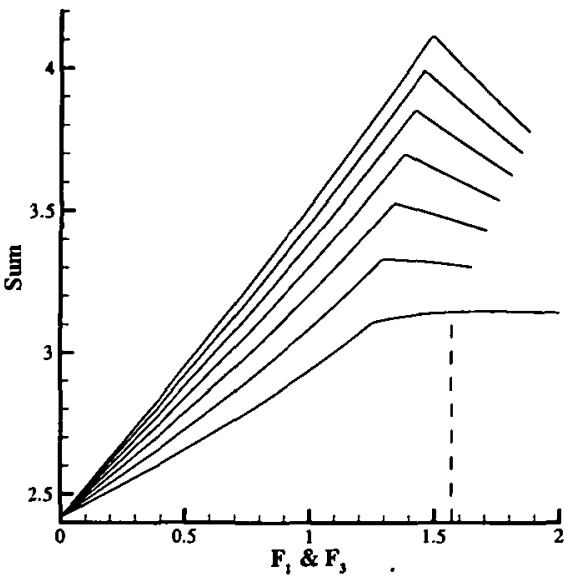

(b)

FIGURE 9. For three sinks of strengths $F_{1}$ at $(-x, 0,1), F_{2}$ at $(0,0,1)$ and $F_{3}$ at $(x, 0,1)$, $x=0.4,0.5, \ldots, 1.0$, (a) feasible regions and (b) sums of sink strengths along the feasible boundary. The $x=0.4$ case is the lowest curve in both cases.

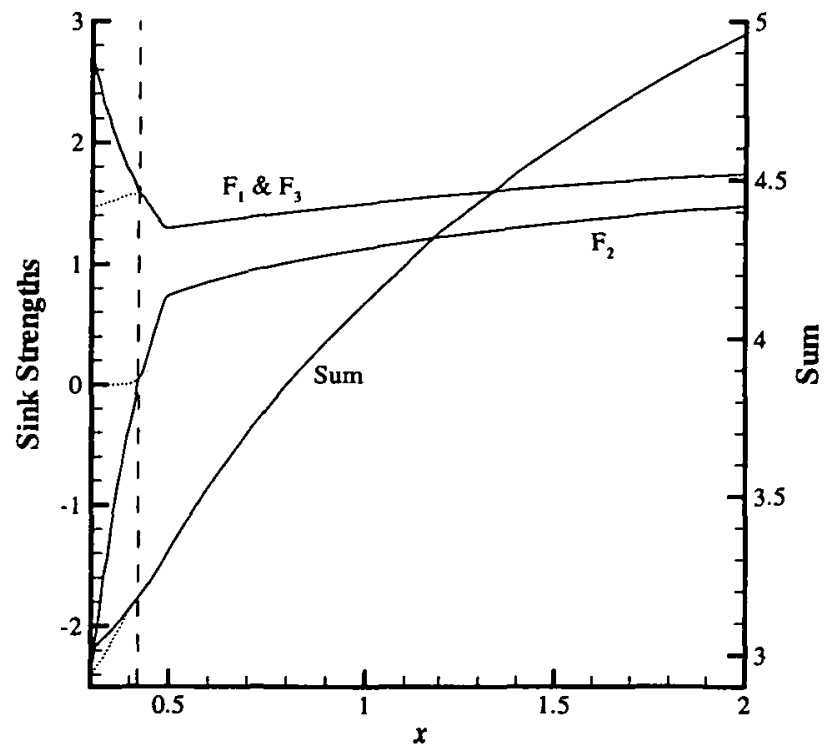

FIGURE 10. For three sinks of strengths $F_{1}$ at $(-x, 0,1), F_{2}$ at $(0,0,1)$ and $F_{3}$ at $(x, 0,1)$, critical flow rates (left axis) and sum of sink strengths (right axis) versus distance between sinks $x$. The dotted extension for small $x$ is when the non-negativity constraint is imposed. 
Figure 8 , for sinks of strength $F_{1}$ at $(-1,0,1), F_{2}$ at $(0,0,1)$ and $F_{3}$ at $(1,0,1)$. With this admittedly coarse mesh, the optimal solution is around $F_{1}=1.5, F_{2}=1.11$, $F_{3}=1.5$. However, we can more easily consider the problem of three sinks of strengths $F_{1}$ at $(-x, 0,1), F_{2}$ at $(0,0,1)$ and $F_{3}$ at $(x, 0,1)$ for some $x$ by assuming $F_{1}=F_{3}$ by symmetry, reducing to a two-parameter problem. Results for the threeparameter problem without using symmetry are identical, but just take longer. Figure 9 shows feasible regions and sums of sink strengths for $x=0.4,0.5, \ldots, 1.0$. As before, we can see the non-smooth behaviour of the feasible boundary, although as $x$ gets smaller and the sinks get closer together the comer becomes less sharp. Of most interest is the curve for $x=0.4$, which has been extended to negative $F_{2}$. This is because from Figure 9 (b), we see that the maximum sum for $x=0.4$ (identified by the vertical dashed line) is not at the corner, but in the negative $F_{2}$ region. And, the sum curve is quite flat near the maximum, indicating the increasingly ill-conditioned nature of the optimisation problem as $x$ gets small.

Figure 10 shows the critical sink strengths as a function of $x$ (left axis) as well as the maximum total flow rate (right axis). The solid lines are the optimal solutions, and the dotted extensions indicate the imposition of the non-negativity constraint. While the overall sum stays smooth, the individual sinks have sudden changes in behaviour. For large $x$ they are smooth, but as $x$ gets small, there is a sudden drop in $F_{2}$, with corresponding jumps in $F_{1}$ and $F_{3}$. If $F_{2}$ is allowed to go negative, $F_{1}$ and $F_{3}$ continue to increase and $F_{2}$ to decrease, but if the non-negativity constraint is imposed there is another change in regime where the middle sink is tumed off and the results become those for two sinks only.

The behaviour of the optimal solution for smaller $x$ seems somewhat nonintuitive; initial expectations were that total flow would always increase if the number of sinks went up, which is clearly not the case here, particularly when we introduce the non-negativity constraint. This behaviour can be explained when we consider what is happening to the interface directly under the middle sink. When the sinks are sufficiently close, the interface under the middle sink is increasingly influenced by the sinks on either side. The outer sinks should be of larger strength than the inner one, since they have no additional sinks outside them to raise the interface further. Once a critical closeness of sinks is achieved, the outer sinks are ideally of a strength to raise the interface directly under them as high as possible, but which causes the interface to be too high between them. The middle sink is then replaced by a source, just large enough to push the interface down to a feasible position below the sinks. With the non-negativity constraint, the middle sink simply turns off, and the best results are obtained with just two sinks.

We should also note that in this case for small $x$, and some later problems, the optimisation problem becomes increasingly ill-conditioned, in the sense that the sum of sinks along the feasible boundary is very flat near the optimal, and we can lose 


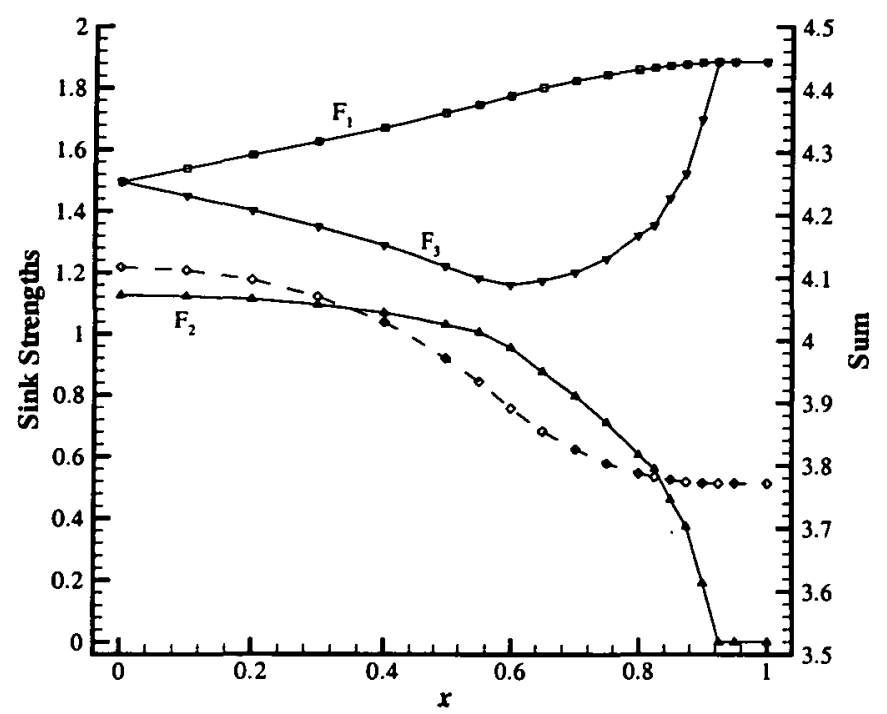

FIGURE 11. For three sinks of strengths $F_{1}$ at $(-1,0,1), F_{2}$ at $(x, 0,1)$ and $F_{3}$ at $(1,0,1)$, critical flow rates (left axis) and sum of sink strengths (right axis) versus the position of middle sink $x$.

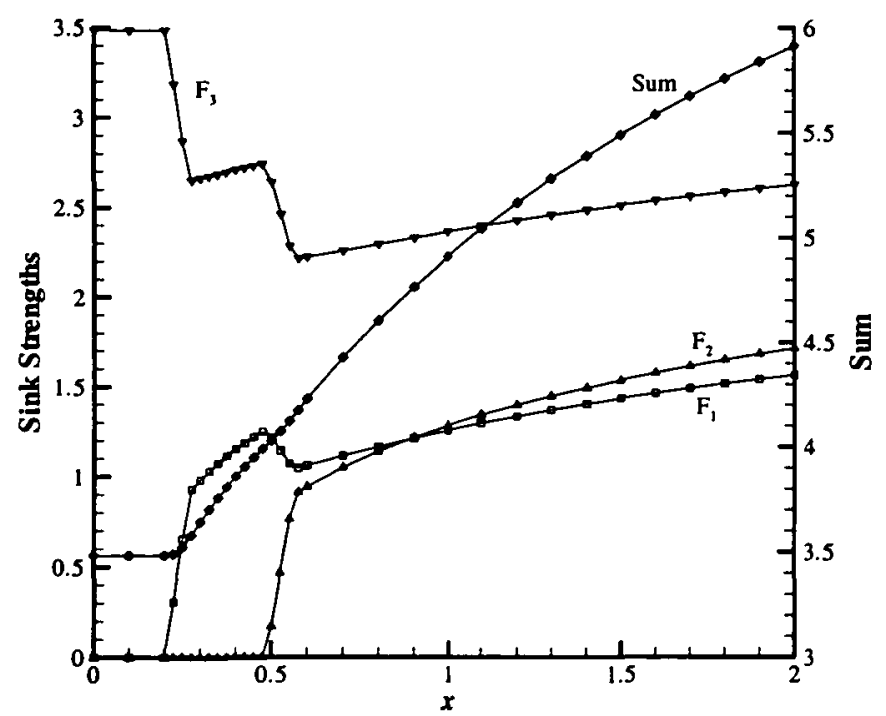

FIGURE 12. For three sinks of strengths $F_{1}$ at $(-x, 0,1), F_{2}$ at $(0,0,1.1)$ and $F_{3}$ at $(x, 0,1.2)$, critical flow rates (left axis) and sum of sink strengths (right axis) versus the distance between sinks $x$. 


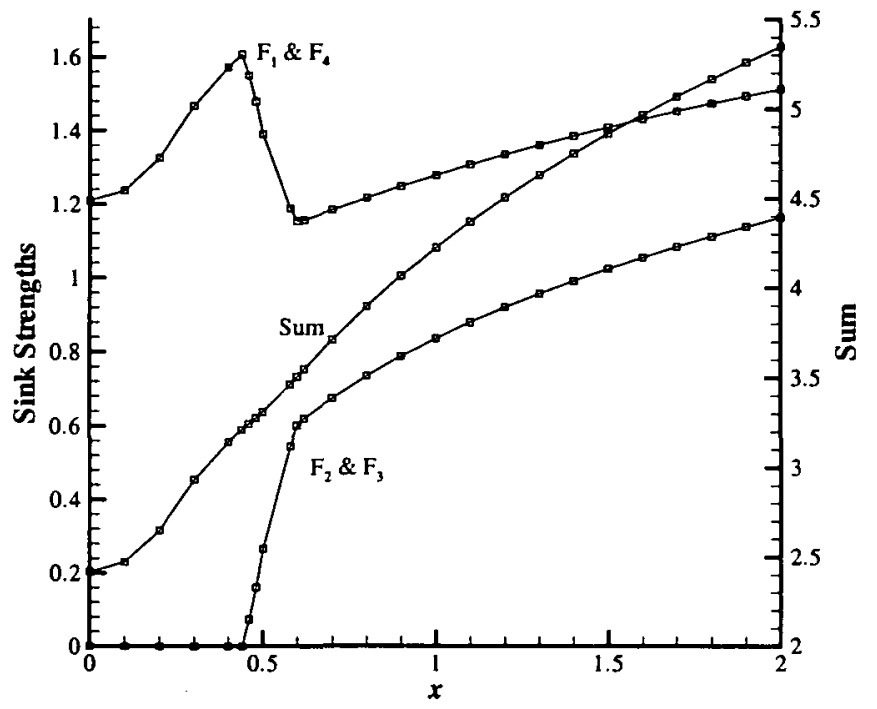

FIGURE 13. For four sinks of strengths $F_{1}$ at $(-x, 0,1), F_{2}$ at $(-x / 3,0,1), F_{3}$ at $(x / 3,0,1)$ and $F_{4}$ at $(x, 0,1)$, critical flow rates (left axis) and sum of sink strengths (right axis) versus $x$.

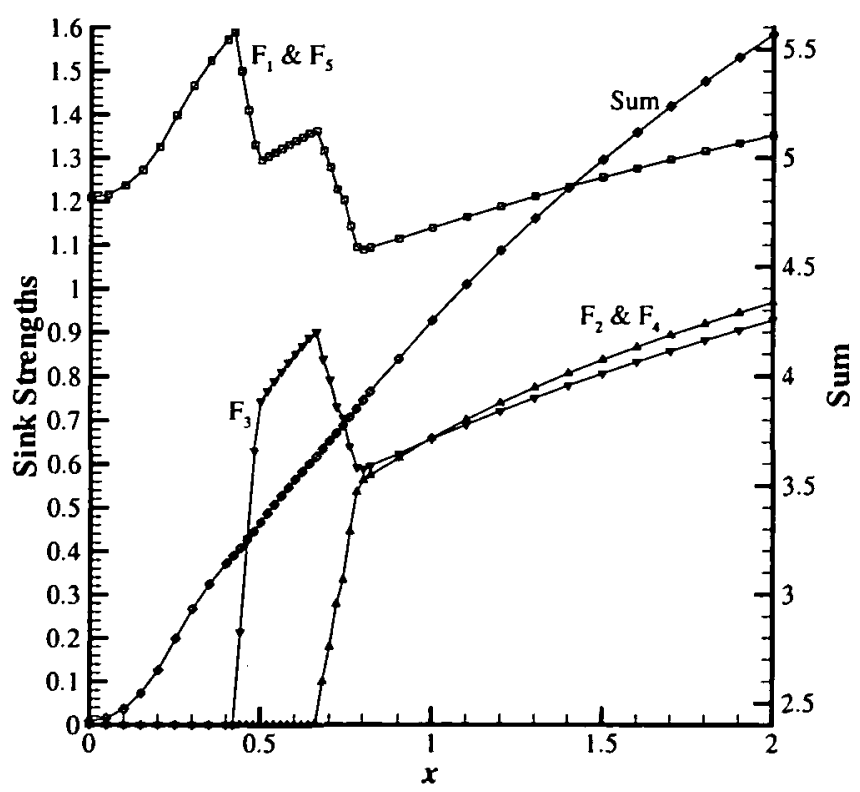

FIGURE 14. For five sinks of strengths $F_{1}$ at $(-x, 0,1), F_{2}$ at $(-x / 2,0,1), F_{3}$ at $(0,0,1), F_{4}$ at $(x / 2,0,1)$ and $F_{5}$ at $(x, 0,1)$, critical flow rates (left axis) and sum of sink strengths (right axis) versus $x$. 
up to four digits of accuracy in the individual sinks. In these situations, we decrease the stopping criteria of the NM method to increase accuracy. We also find that large numbers $(>400)$ of iterations of the NM method are sometimes required, and that as ill-conditioning becomes severe, we need to restart the search algorithm a number of time. This is typical of of the NM method for many ill-conditioned problems, and in fact Press et al. [10] suggest that the NM method should always be restarted at the found calculated optimum to verify that it is in fact truly optimum.

In the remaining results we shall assume the non-negativity constraint is imposed.

5.3.2. Moving middle sink We now consider three sinks of strengths $F_{1}$ at $(-1,0,1), F_{2}$ at $(x, 0,1)$ and $F_{3}$ at $(1,0,1)$ for $x \in[0,1]$. The optimal flow rates and total flow rate as functions of $x$ are shown in Figure 11. Since we are moving the middle sink between the centre and the right sink, the left sink strength $F_{1}$ increases as $x$ increases. The middle sink strength $F_{2}$ reduces as it gets closer to the right sink, and actually turns off for $x>0.925$. Interestingly, the rightmost sink strength $F_{3}$ initially gets smaller as $x$ increases from zero, then starts to increase until the middle sink turns off, when it is identical to $F_{1}$. Again, the total sum is a smooth curve which not surprisingly takes its largest value when $x=0$.

5.3.3. Three sinks at an angle The last three sink example that we include here involves sinks of strengths $F_{1}$ at $(-x, 0,1), F_{2}$ at $(0,0,1.1)$ and $F_{3}$ at $(x, 0,1.2)$. The results as a function of $x$ are shown in Figure 12. Obviously, the higher the sink, the greater the possible flow rate. Reading the graph from the right to the left, as $x$ decreases, the sink strengths decrease smoothly until there is a sudden drop in $F_{2}$ to zero, where both $F_{1}$ and $F_{3}$ increase. Once the middle sink has turned off, the outer sinks return to smoothly decreasing, until the left (lower) sink suddenly drops and the rightmost sink increases in strength. Once the left sink has turned off, we are left with only the upper sink, and so its strength stays constant. Once again, despite the variations in the various sink strengths, the total flow rate stays relatively smooth.

5.4. Four and five sinks in a row In a similar manner as before, we present in Figures 13 and 14 results for four and five sinks evenly spaced between $-x$ and $x$. As we would expect by now, in the four sink case the middle two sinks suddenly disappear at some point where the outer sinks increase in strength. In the five sink case, it turns out that while initially the 2 nd and 4 th sinks are of larger strength than the middle one, with the outer sinks larger still, it is the 2 nd and 4 th sinks that turn off first as $x$ decreases, and then later the middle one leaving the outer sinks only. The total sum continues to be a smooth curve.

5.5. Multiple sinks on an interval The final scenario we wish to consider addresses the problem of concentration of sinks in a different way: given an interval, what is the maximum flow rate possible from equally spaced sinks, and what is the appropriate 
TABLE 1. Sum and individual sink strengths of various numbers of sinks evenly distributed between $(-2,0,1)$ and $(2,0,1)$ inclusive.

\begin{tabular}{|c|c|c|c|c|c|c|c|c|}
\hline No. Sinks & 1 & 3 & 5 & 7 & 9 & 11 & 13 & 15 \\
\hline Sum & 2.418 & 4.961 & 5.568 & 5.785 & 5.873 & 5.907 & 5.921 & 5.938 \\
\hline \multirow[t]{15}{*}{ Ind. Sinks } & \multirow{15}{*}{2.418} & & & & & & & 1.026 \\
\hline & & & & & & & 0.896 & 0.054 \\
\hline & & & & & & 0.925 & 0.356 & 0.457 \\
\hline & & & & & 1.014 & 0.483 & 0.422 & 0.328 \\
\hline & & & & 1.144 & 0.585 & 0.465 & 0.378 & 0.319 \\
\hline & & & 1.351 & 0.731 & 0.550 & 0.439 & 0.367 & 0.318 \\
\hline & & 1.741 & 0.968 & 0.683 & 0.526 & 0.428 & 0.360 & 0.309 \\
\hline & & 1.478 & 0.927 & 0.668 & 0.519 & 0.424 & 0.358 & 0.310 \\
\hline & & 1.741 & 0.968 & 0.683 & 0.526 & 0.428 & 0.360 & 0.309 \\
\hline & & & 1.351 & 0.731 & 0.550 & 0.439 & 0.367 & 0.318 \\
\hline & & & & 1.144 & 0.585 & 0.465 & 0.378 & 0.319 \\
\hline & & & & & 1.014 & 0.483 & 0.422 & 0.328 \\
\hline & & & & & & 0.925 & 0.356 & 0.457 \\
\hline & & & & & & & 0.896 & 0.054 \\
\hline & & & & & & & & 1.026 \\
\hline
\end{tabular}

strength distribution? In Table 1, we detail sink strengths for $n$ sinks equally distributed between the points $(-2,0,1)$ and $(2,0,1)$ as we increase $n$. The results of Table 2 are for sinks between $(-1 / 2,0,1)$ and $(1 / 2,0,1)$. Note that sometimes the sum of the individual sinks is slightly different to the given sum, because results are calculated to higher accuracy, then truncated to three decimal places. In addition, we found that as $n$ increased, the problem became extremely ill-conditioned. For example, with $n=13$ the NM method had to be restarted ten times to finally reach an optimum which did not change to three decimal places. While the total flow did not change appreciably, the individual sink strengths varied by up to $5 \%$ when trying to find the true optimum.

There are a number of interesting observations to be made from these results. Starting with Table 1, with sinks distributed on a relatively large interval, we see that as $n$ increases to about 12 , the pattern is for large sink strengths on the edge, with very similar sink strengths for all sinks in between, although slightly smaller as the sinks get closer to the middle. For 13 and more sinks, we begin to see the same sort of behaviour as for a small number of sinks increasingly closer together. The outer sinks begin to increase in value, and the next sinks in are becoming much smaller. Presumably this sink would turn off as $n$ increased further, and eventually other sinks further in would also disappear. We have not continued further due to increasing ill-conditioning of the problem.

Another observation involves the total flow rates as $n$ increases, which appear to be converging to some maximum flow rate. If we consider the differences between 
TABLE 1 (CONTINUED). Sum and individual sink strengths of various numbers of sinks evenly distributed between $(-2,0,1)$ and $(2,0,1)$ inclusive.

\begin{tabular}{c|ccccccc}
\hline No. Sinks & 2 & 4 & 6 & 8 & 10 & 12 & 14 \\
\hline Sum & 4.182 & 5.348 & 5.701 & 5.838 & 5.894 & 5.915 & 5.930 \\
\hline Ind. Sinks & & & & & & & 0.995 \\
& & & & & & 0.895 & 0.147 \\
& & & & & 0.965 & 0.434 & 0.456 \\
& & & & 1.072 & 0.530 & 0.436 & 0.350 \\
& & & 1.234 & 0.650 & 0.503 & 0.406 & 0.345 \\
& & 1.511 & 0.833 & 0.608 & 0.478 & 0.394 & 0.336 \\
& 2.091 & 1.162 & 0.783 & 0.587 & 0.468 & 0.389 & 0.330 \\
& 2.091 & 1.162 & 0.783 & 0.587 & 0.468 & 0.389 & 0.330 \\
& & 1.511 & 0.833 & 0.608 & 0.478 & 0.394 & 0.336 \\
& & & 1.234 & 0.650 & 0.503 & 0.406 & 0.345 \\
& & & & 1.072 & 0.530 & 0.436 & 0.350 \\
& & & & & 0.965 & 0.434 & 0.456 \\
& & & & & & 0.895 & 0.147 \\
& & & & & & & 0.995 \\
\hline \hline
\end{tabular}

successive flow rates, they reduce by a factor of about 1.5 for $n$ between 4 and 13. For larger $n$, where we see sinks beginning to turn off, the gaps become roughly constant. It seems reasonable to assume that differences between total flow rates would continue to decay after the sink was turned off.

The results in Table 2 are for sinks that are limited to a much smaller interval, and show a completely different behaviour. For an odd number of sinks, where sinks are located at the midpoint of the interval as well as at its endpoints, we see that the optimal solution is in fact for only three sinks. Any additional sinks have no flow to them. For an even number of sinks, we see that only the two outer and most inner sinks have flow, and that the total flow is increasing, although it is less than the total flow for the three sink case. In fact, as $n$ increases and stays even, the inner two sinks become closer to a single sink at the centre. These results indicate that the optimum distribution for sinks on $[-1 / 2,1 / 2]$ is the three sink result. Intervals of width between 1 and 2 would have behaviour between the two extremes shown here.

\section{Conclusion}

This paper has considered the problem of maximising the total flow from a distribution of oil wells without water breakthrough. The Muskat model has been developed as a relatively simple tool that can quickly determine an oil-water interface shape given a set of oil wells and their strengths, or simply establish whether a stable in- 
TABLE 2. Sum and individual sink strengths of various numbers of sinks evenly distributed between $(-1 / 2,0,1)$ and $(1 / 2,0,1)$ inclusive.

\begin{tabular}{c|cccccc}
\hline No. Sinks & 1 & 3 & 5 & 7 & 9 & 11 \\
\hline \hline Sum & 2.418 & 3.331 & 3.331 & 3.331 & 3.331 & 3.331 \\
\hline Ind. Sinks & & & & & & 1.294 \\
& & & & & 1.294 & 0.000 \\
& & & & 1.294 & 0.000 & 0.000 \\
& & & 1.294 & 0.000 & 0.000 & 0.000 \\
& & 1.294 & 0.000 & 0.000 & 0.000 & 0.000 \\
& 2.418 & 0.742 & 0.742 & 0.742 & 0.742 & 0.742 \\
& & 1.294 & 0.000 & 0.683 & 0.000 & 0.000 \\
& & & 1.294 & 0.000 & 0.000 & 0.000 \\
& & & & 1.294 & 0.000 & 0.000 \\
& & & & & 1.294 & 0.000 \\
& & & & & & 1.294 \\
\hline \hline No. Sinks & 2 & 4 & 6 & 8 & 10 & 12 \\
\hline Sum & 3.302 & 3.312 & 3.323 & 3.327 & 3.329 & 3.330 \\
\hline Ind. Sinks & & & & & & 1.282 \\
& & & & & 1.277 & 0.000 \\
& & & & 1.279 & 0.000 & 0.000 \\
& & & 1.300 & 0.000 & 0.000 & 0.000 \\
& & 1.389 & 0.000 & 0.000 & 0.000 & 0.000 \\
& 1.651 & 0.266 & 0.360 & 0.384 & 0.386 & 0.382 \\
& 1.651 & 0.266 & 0.360 & 0.384 & 0.386 & 0.382 \\
& & 1.389 & 0.000 & 0.000 & 0.000 & 0.000 \\
& & & 1.300 & 0.000 & 0.000 & 0.000 \\
& & & & 1.279 & 0.000 & 0.000 \\
& & & & & 1.277 & 0.000 \\
& & & & & & 1.282 \\
\hline \hline
\end{tabular}

terface exists at all. We found that the feasible region of sink strengths leading to a stable interface has a non-smooth boundary, and that the optimal solution lies on this boundary. A constrained (non-negative) version of the Nelder-Mead simplex method, using bisection to search on the boundary of the feasible region, was successfully implemented. While the optimisation problem became increasingly ill-conditioned as oil wells were placed closer together, restarting the NM method successfully led to the optimal solution.

We looked at a variety of sink distributions, motivated by horizontal drilling of oil wells. As well as various detailed results, we have found the initially nonintuitive result that when oil wells are placed too close together, the optimal total flow is obtained when some of the oil wells are not operating. While the individual flow rates 
have sudden changes of behaviour as the oil wells are moved closer together, the total flow stays a remarkably smooth curve.

There are a number of possible extensions of this work. The most obvious is to investigate optimal flow from other potentially interesting distributions of oil wells. While the problems become more ill-conditioned as the number of wells increases, it may be possible to develop a more sophisticated optimisation algorithm than the one used here. If it is sufficiently advanced, it may be possible to use the accurate integral equation solutions of Lucas and Kucera [5] rather than the approximate Muskat method.

\section{Acknowledgements}

I would like to thank Prof. John Blake for introducing me to the Muskat model in an undergraduate vacation project in 1988, and Theo Frederiks, a research student who did some initial work with me on optimisation using SQP for this problem in 1999. I would also like to thank the referees for their useful comments.

\section{References}

[1] J. Bear, Dynamics of fluids in porous media (McGraw-Hill, New York, 1972).

[2] J. R. Blake and A. Kucera, "Coning in oil reservoirs", Math. Scientist 13 (1988) 36-47.

[3] J. Gunning, L. Paterson and B. Poliak, "Coning in dual completed systems", J. Pet. Sci. Eng. 23 (1999) 27-39.

[4] S. K. Lucas, J. R. Blake and A. Kucera, "A boundary-integral method applied to water coning in oil reservoirs", J. Austral. Math. Soc. Ser. B 32 (1991) 261-283.

[5] S. K. Lucas and A. Kucera, "A 3D boundary integral method applied to the water coning problem", Physics of Fluids 8 (1996) 3008-3022.

[6] MathWorks Inc., Matlab, the language of technical computing, 1994-2001, http: / / www . mathworks . com/.

[7] K. I. M. McKinnon, "Convergence of the Nelder-Mead simplex method to a nonstationary point", SIAM J. Optim. 9 (1998) 148-158.

[8] M. Muskat, Physical principles of oil production (McGraw-Hill, New York, 1949).

[9] M. Muskat and R. D. Wyckoff, "An approximate theory of water coning in oil production", Trans. AIME 114 (1935) 144-159.

[10] W. H. Press, S. A. Teukolsky, W. T. Vetterling and B. P. Flannery, Numerical recipes in Fortran 77, 2nd ed. (Cambridge University Press, Cambridge, 1992).

[11] C. J. Price, I. D. Coope and D. Byatt, "A convergent variant of the Nelder-Mead algorithm", $J$. Optim. Theory Appl. 113 (2002) 5-19.

[12] H. Zhang, D. A. Barry and G. C. Hocking, "Analysis of continuous and pulsed pumping of a phreatic aquifer", Adv. Wat. Res. 22 (1999) 623-632.

[13] H. Zhang and G. C. Hocking, "Axisymmetric flow in an oil reservoir of finite depth caused by a point sink above an oil-water interface", J. Engrg. Math. 32 (1997) 365-376. 